\title{
The tunnelling of electromagnetic radiation in chirped dielectric structure
}

\author{
Anatoliy Kozar, Vladimir Marchenko, and Pavel Shestakov* \\ Lomonosov Moscow State University, Physics Department, Leninskie Gory, Moscow, 119991 Russia
}

\begin{abstract}
The tunnelling of optical beams through chirped Bragg grating is considered. Lateral shifts of transmitted and reflected beams were calculated. The saturation of Goos-Hanchen shift of transmitted light beams is shown.
\end{abstract}

The chirped Bragg grating $(\mathrm{CBG})$ is the structure with linear deviation of its period along propagation direction:

$$
d(z)=d_{0}+\alpha z\left(d_{0}-\text { grating period, } \alpha-\text { deviation parameter }\right)
$$

The use of CBG for dispersion compensation and amplification of chirped pulses is based on its reflective properties [2]. When we deal with spatial beams a CBG allows obtaining after reflection either divergent or convergent light beams. The tunnelling of optical radiation decreases an efficiency of a CBG since it leads to power loss in reflection. Along with that the transmission of electromagnetic radiation through such structures allow to model quantum effects, which direct experimental observation is not available so far $(3,4)$.

It is well known that CBGs have local forbidden gaps not only in frequency and spatial spectra but also along the longitudinal coordinate - so called photonic barrier (PB). The PB boundaries are determined from the expression $\delta= \pm \kappa$, where the detuning from the Bragg resonance $\delta=k_{0} \varepsilon_{0}{ }^{I / 2} \cos \theta_{0}-\pi / d_{0}+\beta\left(z-z_{0}\right), \kappa=\Delta \varepsilon / 4 k_{0} \varepsilon_{0}{ }^{l / 2}$ - the Bragg coupling, $k_{0}=\pi / d_{0}, \beta=$ $-\alpha \pi /\left(2 n_{0} d_{0}^{2}\right), \varepsilon_{0}=n_{0}{ }^{2}, \Delta \varepsilon \approx\left(\varepsilon_{1}-\varepsilon_{2}\right) / 2, \Delta \varepsilon / \varepsilon_{0}<<1$ - the dielectric contrast, $n_{0}$ - the average refractive index. The length of the $\mathrm{PB}$ is determined by the formula $l_{P B}=2 \kappa /|\beta|$.

Fig. 1 shows the oblique incidence $\left(\theta_{0}=30^{\circ}\right)$ of a weakly diverging beam with gaussian profile under the conditions when the photonic barrier with $l_{P B}=8 \mu \mathrm{m}$ is located in the $L / 2$ vicinity. There is virtually no profile distortion and phase modulation of the light beam. The total lateral shift (segment $\mathrm{CD}=35 \mu \mathrm{m}$ ) of the reflected beam at the boundary $z=0$ is composed of a shift due to the beam propagation in the transparency region of the chirped structure and a shift at the photonic barrier surface $(A B=2 \mu \mathrm{m})$, or the Goos - Hänchen shift.

The oblique incidence of a light beam on a finite periodic structure without period deviation $(\alpha=0)$ is accompanied by the Goos-Hänchen shift of reflected and transmitted beams when the angular spectrum of the beam is within the band gap [5]. Since the phase of the reflection coefficient $\phi_{R}=\arctan (\delta / q \tanh \kappa L)$ the Goos-Hanchen shift is determined by $\Delta_{R}=d \phi_{R} / d k_{x}=\tanh (\kappa L) / \kappa \tan \theta$ when $\delta=0$, where $q=\left(\delta^{2}-\kappa^{2}\right)^{1 / 2}$. Assuming that the light reflects not from the surface of the periodic structure but from the effective depth $\left(d_{\text {eff }}=1 / 2 \kappa\right)$ inside this structure we simplify the expression for the Goos-Hänchen shift as $\Delta=2 d_{\text {eff }} \tan \theta$.

These estimations are valid for semi-infinite structures, i.e. the attenuation inside the PB should be sufficient for the reflection coefficient is about unity. Because of this the length of

*Corresponding author: iveage@physics.msu.ru 
the $\mathrm{PB}$ is restricted by the condition $\tan \left(\kappa l_{P B}\right) \approx 1$, which means that $\kappa l_{P B} \geq 3$. When a light beam is incident onto a structure with $\kappa l_{P B}<3$, a noticeable tunnelling of electromagnetic radiation through the PB is observed. This regime reduces the efficiency of the compressionstretching mechanism of reflected pulses (beams).

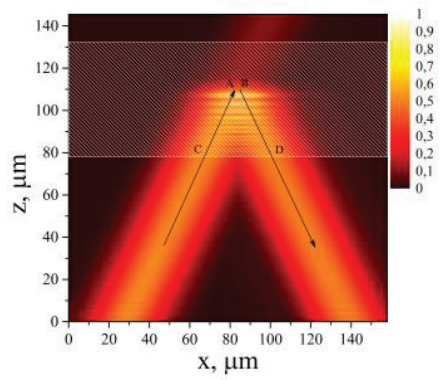

Fig. 1. Reflection of a wide light beam from a photonic barrier $\left(l_{P B}=8 \mu \mathrm{m}\right)$ located inside the periodic structure $\left(\alpha=2 \cdot 10^{-3}, n_{1}=1.6, n_{2}=1.4, \theta_{0}=30^{\circ}\right)$. The arrows indicate the direction of beam propagation.

The value of the Goos-Hänchen shift of a transmitted beam is estimated numerically by the expression:

$$
\Delta_{T}=\int x\left|E_{t r}\right| d x / \int\left|E_{t r}\right| d x
$$

where $E_{t r}$ - the amplitude of forward propagating wave, $x$ - the traverse coordinate. The Fig. 2 shows the calculated values of the Goos-Hänchen shift in dependence on periodic structure for the different values of photonic barrier lengths located in the $L / 2$ vicinity.

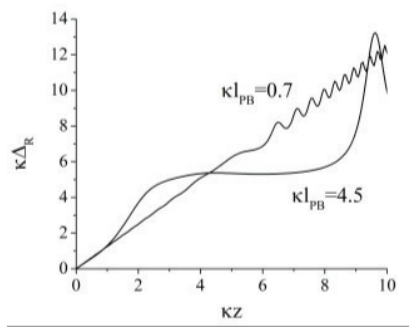

Fig. 2. The values of the Goos-Hänhen shift of a transmitted beam in dependence on CBG length $(\alpha=$ $\left.2 \cdot 10^{-7}, L=2 \mathrm{~cm}, n_{0}=1.4, \Delta \varepsilon / \varepsilon_{0}=10^{-3}\right)$.

When $\kappa l_{P B}<3$ the shift of a transmitted beam increases linearly with length of the structure. If the length of the photonic barrier is quite large $\left(\kappa l_{P B}>3\right)$ the values of the GoosHänchen shift saturates. This effect is analogous to the time delay saturation of tunnelled pulses through a periodic structure without a period deviation $(\alpha=0)$. It was noted that the saturation of time delay happens because of energy storage in a near-surface layer of the periodic structure [6].

This study is supported by the Russian Science Foundation (Grant No 17-11-01157).

\section{References}

[1] S. Kaim, S. Mokhov, B. Y. Zeldovich, L.B. Glebov, Opt. Eng. SPIE 5, 53 (2014)

[2] R. Sun, D. Jin, F. Tan et al., Opt. Express 24, 20 (2016)

[3] S. Longhi, Phys. Res. Int. 2010, 645106 (2010)

[4] P.B. Wilkinson, Phys. Rev. E 65, 5 (2002)

[5] D. Felbaq, A. Moreau, R. Smaâli, Opt. Lett. 28, 18 (2003)

[6] H. Winful, Opt. Express 10, 25 (2002) 